\title{
Management of Chronic Inflammatory Gingival Enlargement associated with Orthodontic Therapy: A Case Report
}

\author{
Raghaveena Ingle, P. Raja Babu, S. Vidya Sagar, Suryakanth Malgikar \\ Department of Periodontics, Kamineni Institute of Dental Sciences, Narketpally, Nalgonda, Telangana, India
}

Email for correspondence: dr.raghaveena@gmail.com

\begin{abstract}
Many adults who seek routine restorative dentistry have problems with malposition of the teeth, which not only compromise their ability to clean and maintain their dentitions but also help to maintain the health of periodontium. Recently, a greater focus on dentofacial esthetics in the adult population is seen with an increasing demand for orthodontic treatment in appearance-conscious adults. Sometimes, during orthodontic treatment, fixed appliances that bond the teeth surface could cause problem toward periodontal tissues making it difficult to control the plaque, especially in the marginal region that could cause inflammation and gingival enlargement (GE). GE due to the local irritation of the plaque can be treated with plaque control, scaling, root planing, until surgical intervention. Treatment with surgical intervention for GE is to eliminate the gingival pocket, so the patient can easily clean the teeth, thereby slowing down the disease progression and retain the normal gingival status again. The present case report presents management of chronic GE associated with orthodontic therapy.
\end{abstract}

Key words: Gingivectomy, gingival overgrowth, orthodontic treatment

\section{INTRODUCTION}

Gingival enlargement (GE) is a multifactorial condition that develops in response to various stimuli and interactions between the host and the environment. This increase in the size of the gingiva may be plaque-induced or associated with systemic hormonal disturbances. It also occurs as a manifestation associated with genetic disorders or may be due to several blood dyscrasias, such as leukemia and thrombocytopenia. ${ }^{[1]}$ Based on the extent and severity, these enlargements may lead to functional disturbances such as altered speech, difficulty in mastication, and esthetic and psychological problems. Inflammatory GE may be

\section{Quick Response Code Article Info:}

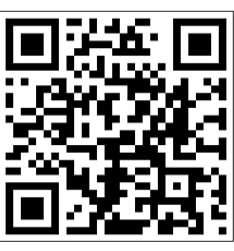

doi: $\mathbf{1 0 . 5 8 6 6 / 2 0 1 7 . 9 . 1 0 1 8 7}$

Received: 05-08-2017

Revised: 07-09-2017

Accepted: 22-09-2017

Available Online: 27-11-2017 (www.nacd.

in)(C) NAD, 2017 - All rights reserved categorized as acute or chronic. Chronic changes are being much more common. ${ }^{[1]}$ The ability to perform oral hygiene measures is compromised in some patients with GEs, which may be further complicated by the presence of prosthesis and fixed orthodontic appliances. This may lead to more inflammation and further plaque accumulation leading to transformation of the gingival sulcus into a periodontal pocket where plaque removal becomes impossible. The therapeutic approaches related to GE are based on the underlying etiology and the subsequent changes it manifests on the tissues. The prime treatment modalities involve obtaining a detailed medical history and non-surgical periodontal therapy, followed by surgical excision to retain esthetical and functional demands. The present case report presents the management of chronic GE associated with orthodontic therapy.

\section{CASE REPORT}

A21-year-old systemically healthy female patient reported to the Department of Periodontology with a chief complaint of swollen gums in the upper and lower front teeth region of the jaws. The patient also 
experienced bleeding from the gums occasionally while brushing her teeth. Dental history revealed that she was undergoing orthodontic treatment for the pathological migration of maxillary anterior teeth since 6 months.

Intraoral examination revealed orthodontic molar tubes and brackets in maxillary teeth. Diffused gingival overgrowth involving marginal, papillary, and attached gingiva in the maxillary and mandibular arches was shown in Figure 1. Gingival overgrowth was more prominent in the anterior sextants of the jaw, especially on the maxillary palatal aspect which was soft and edematous in consistency [Figure 2]. Bleeding on probing was generalized through all the dentition. Initial periodontal therapy with scaling and root planing using the hand and ultrasonic instrumentation followed by a gingivectomy procedure was planned to improve esthetics and function.

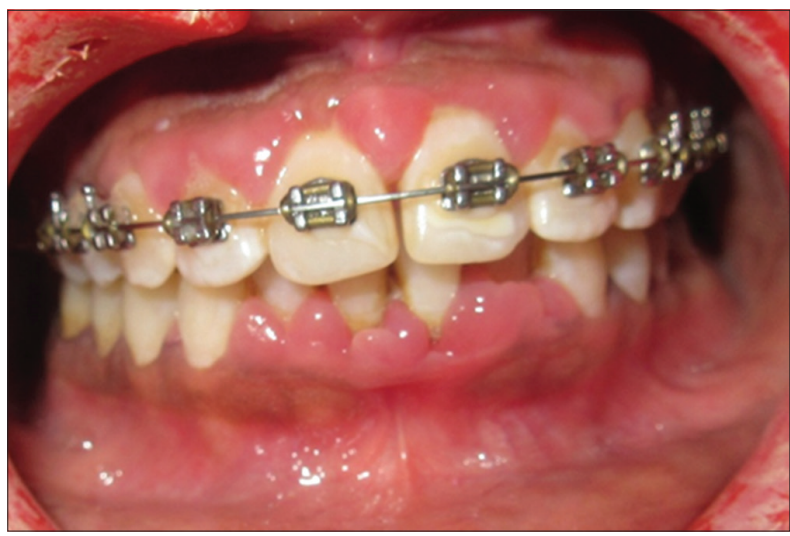

Figure 1: Clinical picture showing gingival enlargement of maxillary and mandibular anterior teeth

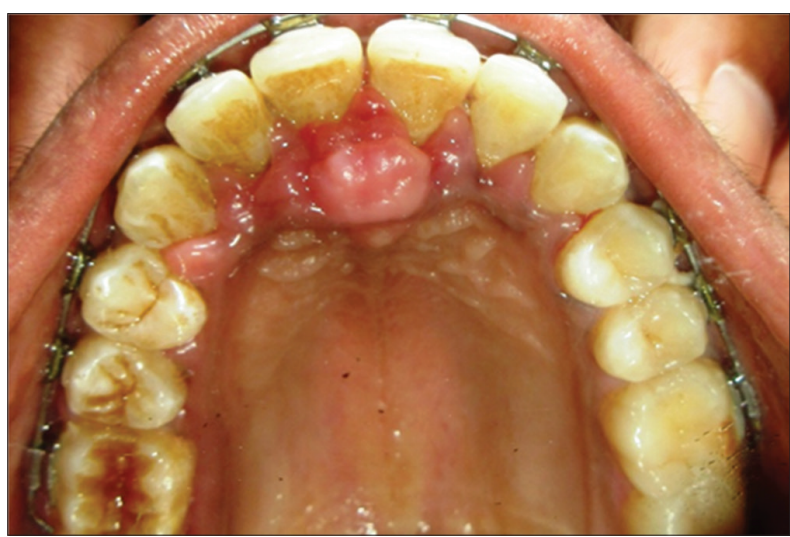

Figure 2: Pre-operative photograph showing gingival overgrowth in the maxillary palatal region of the jaw

\section{Pre-surgical therapy}

Supragingival and subgingival scaling and root planing were performed using the hand and ultrasonic instrumentation.

\section{Surgical procedure}

Four weeks after scaling and root planning, gingivectomy was planned in the maxillary palatal anterior region. After administration of local anesthesia, external bevel incision was given with Kirkland knife directed at the base of the pocket [Figure 3]. Excised tissue was sent for histopathological examination [Figure 4]. This procedure heals by secondary intention and does not provide access to the underlying bone [Figure 5]. Periodontal dressing was given at the site of the surgery [Figure 6]. The patient was advised antibiotics for 1 week postoperatively amoxicillin $500 \mathrm{mg}$ TID and nonsteroidal antiinflammatory drug (Hifenac P BID, 3 days). Oral hygiene instructions were given, and the patient was advised to use chlorhexidine mouthwash $(0.2 \%$

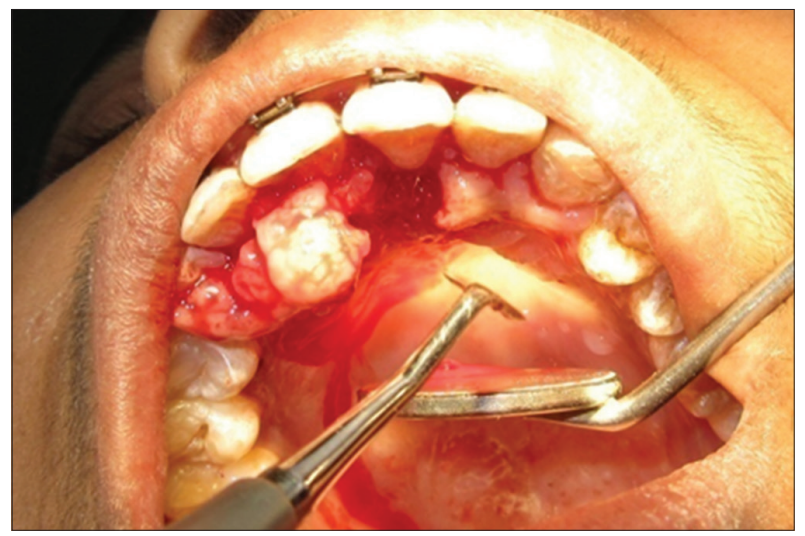

Figure 3: External bevel gingivectomy

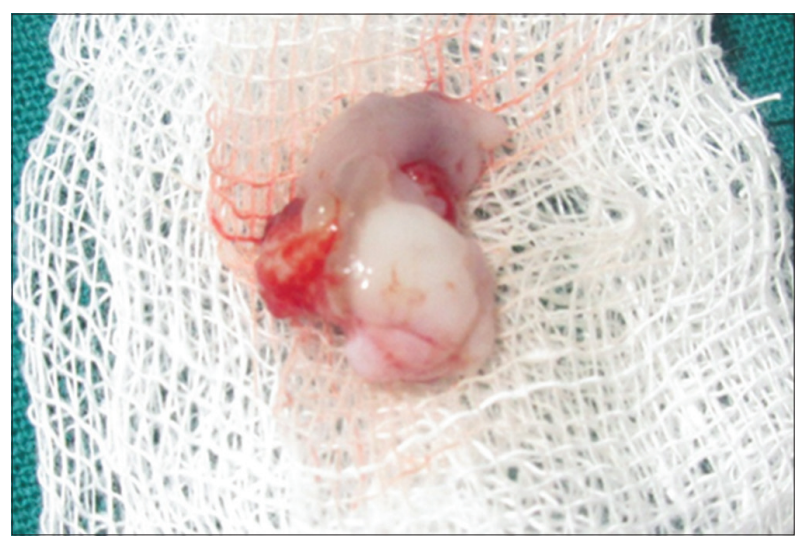

Figure 4: Photograph showing excised tissue 


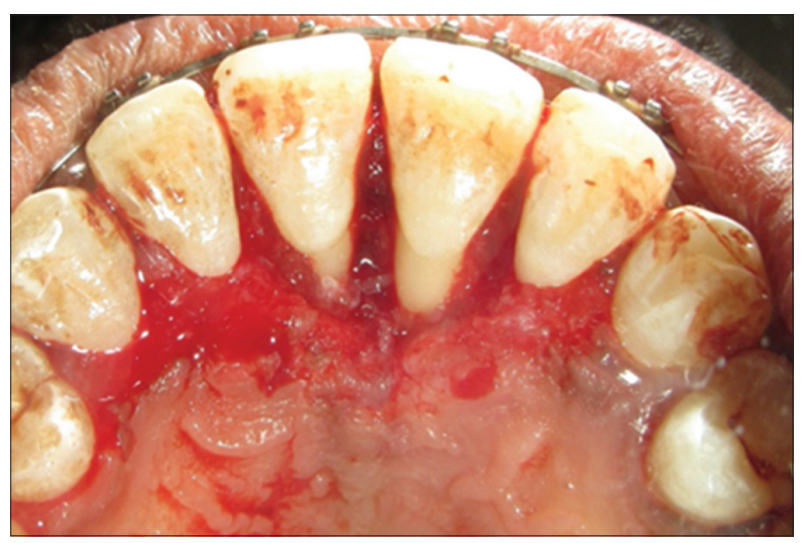

Figure 5: Immediate post-operative picture

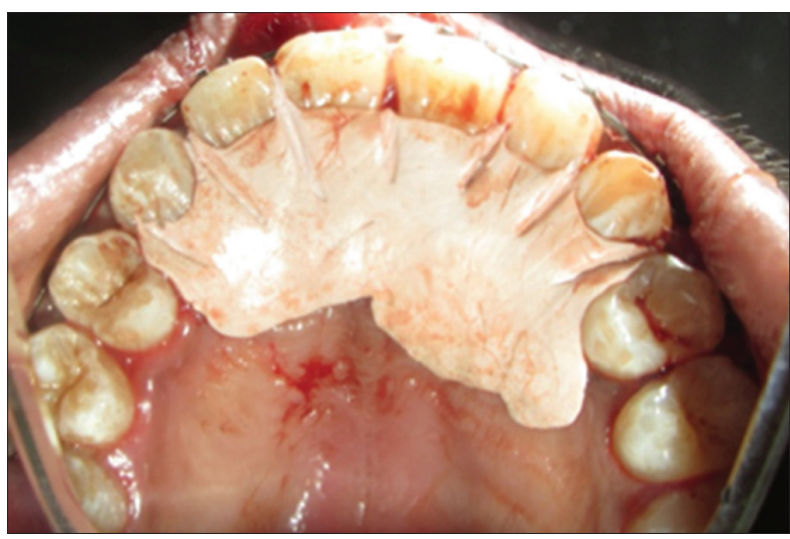

Figure 6: Periodontal dressing

Clohex $^{\mathrm{TM}}$, Dr. Reddy's Laboratories Ltd., India) twice daily for 1 week. The histopathological examination revealed the presence of collagen fiber bundles with moderate chronic inflammatory cell infiltrate with engorged and dilated blood vessels in the connective tissue stroma [Figure 7].

One month postoperatively, intraoral examination revealed that the maxillary surgical site healed satisfactorily [Figure 8]. There was no recurrence of the GE in the maxillary anterior sextant. Initial periodontal therapy was performed again, and oral hygiene instructions were reinforced. The patient was also counselled regarding the importance of follow-up and maintenance with special emphasis on motivation.

\section{DISCUSSION}

GE is defined as an abnormal overgrowth of gingival tissues. It is an unusual condition causing esthetic, functional, and psychological disturbance in an individual. If the cause is clearly evident, it may be easy for a dentist to arrive at a clinical

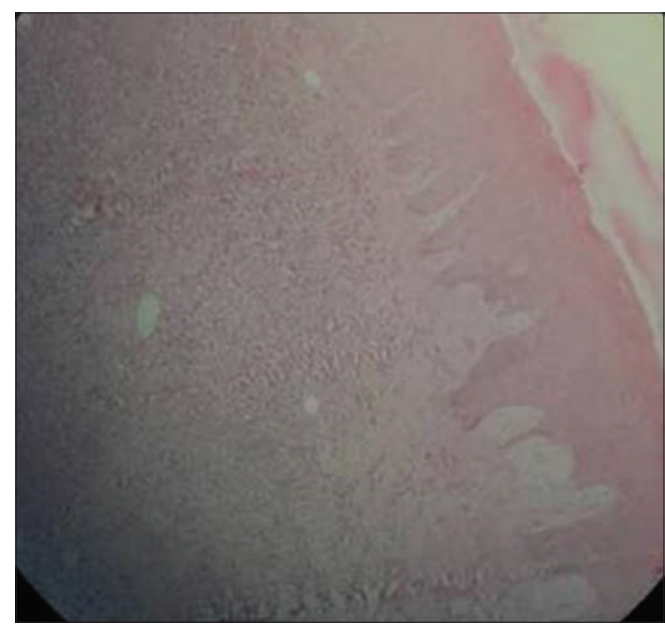

Figure 7: $\mathrm{H}$ and E stained section showing hyperplastic gingiva and inflamed connective tissue

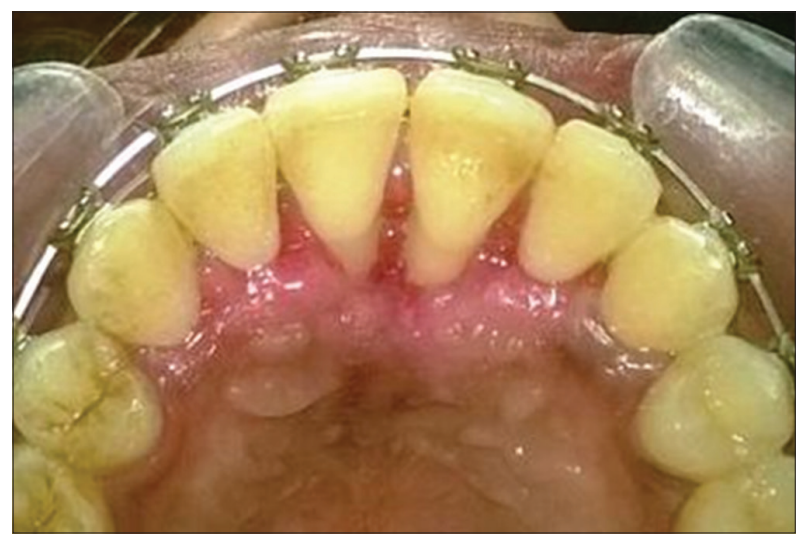

Figure 8: Clinical picture showing satisfactory healing at the maxillary surgical site after 1 month

diagnosis of $\mathrm{GE}$, but at times, it becomes necessary to seek medical advice to explore the cause and identify the underlying diseases, drug interactions or the natural body changes, and to develop an effective treatment plan. Enlargement of the gingiva resulting from acute or chronic inflammatory changes is usually a secondary complication to any other type of enlargement, and this results in a combined enlargement. Hence, understanding the double etiology is the key to successful treatment outcome. This originates as a slight ballooning of the interdental papilla and marginal gingiva forming a bulge around the involved tooth. It may be localized or generalized and progress slowly unless complicated by acute infection or trauma. They are usually painless, slow-growing masses and may occasionally occur as sessile or pedunculated mass resembling a tumor. They may 
undergo a spontaneous reduction in size, followed by exacerbation and continued enlargement. They also have a preponderance of cells and fluid, with vascular engorgement, new capillary formation, and associated degenerative changes, thereby showing exudative and proliferative features of chronic inflammation. Lesions that are relatively firm and pink and resilient have a greater fibrotic component with an abundance of fibroblasts and collagen fibers. They are caused by prolonged exposure to dental plaque, poor oral hygiene, irritation by anatomic abnormalities, and improper restorative, and orthodontic appliances are the factors which favor plaque accumulation and increase the pathogenicity of the microbes. ${ }^{[2]}$ Chronic inflammatory enlargements caused principally by edema and cellular infiltration are treated by scaling and root planing, provided the size of enlargement does not interfere with complete removal of deposits. When they include a fibrotic component, then surgical removal is the treatment of choice. When enlarged gingiva remains soft and friable, a gingivectomy is used. However, if gingivectomy removes all of the attached gingiva, creating mucogingival problems, then flap operation is indicated. ${ }^{[3]}$ The present case report reveals chronic inflammatory GE due to orthodontic therapy. These enlargements are often associated with a long-standing bacterial plaque accumulation. Furthermore, the presence of the appliances may have further compromised the maintenance of adequate oral hygiene. Consequently, it was noticed that, once the appliances were removed and oral hygiene instructions were reinforced, the patient was able to maintain good oral hygiene. A study by Sallum et al. showed a significant impact of orthodontic appliance removal and professional prophylaxis on periodontal health. ${ }^{[4]}$ Reinforcement of effective oral hygiene is essential since patients have a tendency to revert to their original behavior. This reflects the importance of patient education, motivation, and compliance during and after dental treatment. The patient must be placed into a maintenance schedule to preserve a healthy dentition. A systematic review by Gray and Mc Intyre determined the effectiveness of orthodontic oral health promotion (OHP) on gingival health, and it has been found that OHP program for patients undergoing fixed appliance orthodontic treatment produces a short-term reduction (up to 5 months) in plaque and improvement in gingival health. ${ }^{[5}$ Current studies have shown that oral hygiene is strongly related to the gingiva inflammation and reversibility associated after scaling, curettage, and root planning interventions. ${ }^{[6-9]}$ This reflects the importance of patient education, motivation, and compliance during and after dental treatment.

\section{CONCLUSION}

In the present case report, it could be inferred that the orthodontic treatment, especially fixed appliance, could cause plaque accumulation resulting from the difficulty of the patient in plaque control, eventually allowing gingiva inflammation to occur. Meticulous oral hygiene measures during orthodontic treatment course significantly would optimize the outcome and prevent adverse events on periodontium.

\section{REFERENCES}

1. Carranza FA, Hogan EL. Gingival enlargement. Newman Takei Carranza. Carranza's Clinical Periodontology. $10^{\text {th }}$ ed Philadelphia, PA: Elsevier; 2009. p. 373-90.

2. Hirschfeld I. Hypertrophic gingivitis: Its Clinical Aspect. J Am Dent Assoc 1932;19:799.

3. Jadhav T, Bhat KM, Bhat GS, Varghese JM. Chronic inflammatory gingival enlargement associated with orthodontic therapy - Acase report. J Dent Hyg 2013;87:19-23.

4. Sallum EJ, Nouer DF, Klein MI, Gonçalves RB, Machion L, Wilson Sallum A, et al. Clinical and microbiologic changes after removal of orthodontic appliances. Am J Orthod Dentofacial Orthop 2004;126:363-6.

5. Gray D, McIntyre G. Does oral health promotion influence the oral hygiene and gingival health of patients undergoing fixed appliance orthodontic treatment? A systematic literature review. J Orthod 2008;35:262-9.

6. Lang NP, Cumming BR, Löe H. Toothbrushing frequency as it relates to plaque development and gingival health. $J$ Periodontol 1973;44:396-405.

7. Yanover L, Ellen RP. A Clinical and microbiological examination of gingival disease in prepubescent females. $J$ Periodontol 1986;57:562-7.

8. Moore WE, Holdeman LV, Smibert RM, Good IJ, Burmeister JA, Palcanis KG, et al. Bacteriology of experimental gingivitis in young adult humans. Infect Immun 1982;38:651-67.

9. Mousquès T, Listgarten MA, Phillips RW. Effect of scaling and root planing on the composition of the human subgingival microbial flora. J Periodontal Res 1980;15:144-51. 